\title{
Quantum interference of two photons emitted from a luminescence center in GaAs:N
}

Michio Ikezawa, Liao Zhang, Yoshiki Sakuma, and Yasuaki Masumoto

Citation: Appl. Phys. Lett. 110, 152102 (2017); doi: 10.1063/1.4979520

View online: http://dx.doi.org/10.1063/1.4979520

View Table of Contents: http://aip.scitation.org/toc/apl/110/15

Published by the American Institute of Physics

\section{Articles you may be interested in}

Independent tuning of excitonic emission energy and decay time in single semiconductor quantum dots

Appl. Phys. Lett. 110, 151102151102 (2017); 10.1063/1.4979481

Experimental prototype of a spin-wave majority gate

Appl. Phys. Lett. 110, 152401152401 (2017); 10.1063/1.4979840

Bright narrowband biphoton generation from a hot rubidium atomic vapor cell

Appl. Phys. Lett. 110, 161101161101 (2017); 10.1063/1.4980073

Optical probing of the Coulomb interactions of an electrically pumped polariton condensate

Appl. Phys. Lett. 110, 151103151103 (2017); 10.1063/1.4979836

Electrically driven and electrically tunable quantum light sources

Appl. Phys. Lett. 110, 071102071102 (2017); 10.1063/1.4976197

Ferroelectric, pyroelectric, and piezoelectric properties of a photovoltaic perovskite oxide

Appl. Phys. Lett. 110, 063903063903 (2017); 10.1063/1.4974735

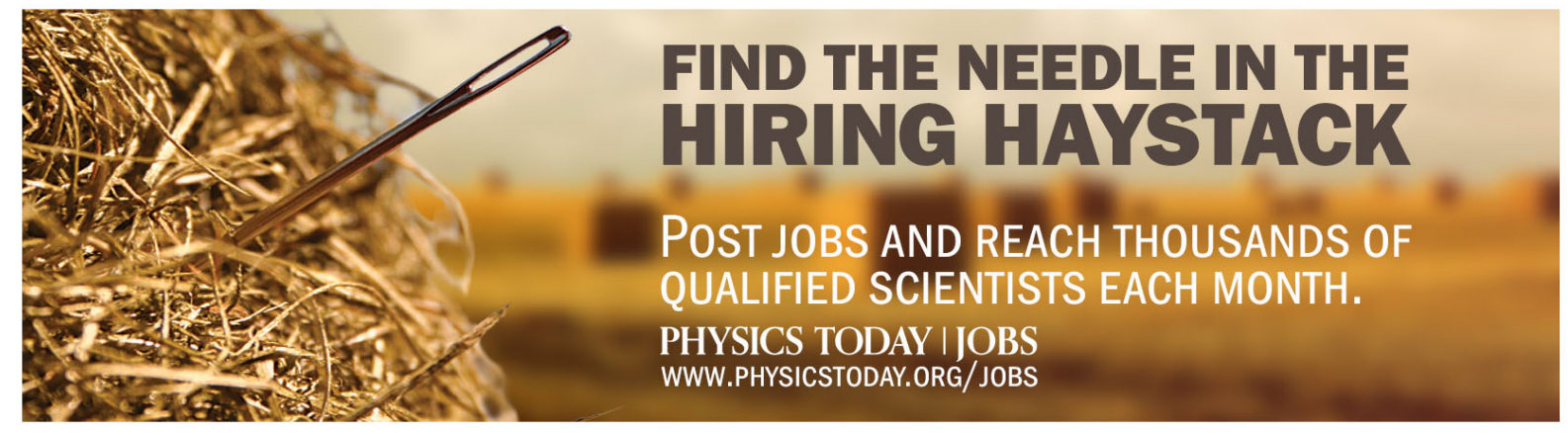




\title{
Quantum interference of two photons emitted from a luminescence center in GaAs:N
}

\author{
Michio Ikezawa, ${ }^{1}$ Liao Zhang, ${ }^{1}$ Yoshiki Sakuma,${ }^{2}$ and Yasuaki Masumoto ${ }^{1}$ \\ ${ }^{1}$ University of Tsukuba, 1-1-1 Tennoudai, Tsukuba, Ibaraki 305-8571, Japan \\ ${ }^{2}$ National Institute for Materials Science, 1-1 Namiki, Tsukuba, Ibaraki 305-0044, Japan
}

(Received 10 January 2017; accepted 13 March 2017; published online 11 April 2017)

\begin{abstract}
The indistinguishability of photons emitted from a nitrogen luminescence center in GaAs is investigated by two-photon interference under nonresonant optical excitation. A clear dip is observed in a parallel polarization configuration for consecutively emitted two photons with a 2-ns time interval. The indistinguishability is approximately 0.24 , and is found to be independent of the time interval between $2 \mathrm{~ns}$ and $4 \mathrm{~ns}$. These results suggest the existence of a very fast dephasing mechanism within 2 ns. Published by AIP Publishing. [http://dx.doi.org/10.1063/1.4979520]
\end{abstract}

Indistinguishable photons are essential prerequisites for realizing advanced quantum information processing schemes like quantum teleportation ${ }^{1-3}$ and linear-optic quantum computation. $^{4,5}$ A high degree of indistinguishability requires almost complete wave-packet overlap in energy, space, time, and polarization. So far, many kinds of single photon emitters, for example, single atoms, ${ }^{6}$ trapped ions, ${ }^{7}$ molecules, ${ }^{8}$ nitrogen-vacancy centers in diamond, ${ }^{9,10}$ and semiconductor quantum dots (QDs), ${ }^{11,12}$ have been demonstrated to generate indistinguishable photons through two-photon interference (TPI) experiments.

From an application standpoint, semiconductor-based solid-state single photon sources, like QDs, have been considered to be the most feasible candidates for realizing integrated devices for quantum information technology because of their associated advantages, such as robustness, integrability, and possibility for electrical drive ${ }^{13}$ However, to obtain indistinguishable photons from two independent (isolated) photon sources in the solid-state, the energy mismatch between two emitters, arising from an inhomogeneity of the emitter itself or their environment in the solid-state, must be compensated by some extrinsic methods such as thermal modulation, ${ }^{14}$ strain, ${ }^{15}$ or electrical tuning. ${ }^{16}$ Therefore, obtaining indistinguishable photons from more than two independent emitters is still a challenging task for solid-state single photon sources. Compared with QDs, impurity luminescence centers in semiconductors would be superior in terms of scalability, because well-defined emission energy is expected from such luminescence centers, at least in principle. In fact, they often exhibit sharp luminescence spectra even in ensemble measurements. A pioneer study of TPI by using a fluorine donor impurity in $\mathrm{ZnSe} / \mathrm{ZnMgSe}$ has been demonstrated under a nonresonant excitation scheme. ${ }^{17}$

Recently, a nitrogen impurity center in III-V compound semiconductors has shown potential as a novel single photon source. ${ }^{18-20}$ Nitrogen in these systems is known as a typical isoelectronic impurity, and their optical properties have been studied extensively by macro photoluminescence (PL) $)^{21-23}$ and micro PL method. ${ }^{24-26}$ These isoelectronic centers show sharp and bright luminescence peaks below the bandgap energy, and are useful as single photon sources. In fact, single photon emission with well-defined energy has been demonstrated for nitrogen impurity centers in $\mathrm{GaP}^{18}$ and GaAs. ${ }^{20}$ In particular, a nitrogen impurity in GaAs is important for indistinguishable photon generation, because it has a higher radiative decay rate, which is favorable for satisfying the Fourier-transform limited coherence time required for indistinguishable photon generation. The indistinguishability of photons emitted from these isoelectronic centers has not been examined so far.

In this work, we have investigated the indistinguishability of photons emitted from a single nitrogen impurity center in GaAs by Hong-Ou-Mandel type TPI measurements under a nonresonant excitation scheme. We show the indistinguishability of approximately $24 \%$ from a center excited by pulse pairs with a time separation of $2 \mathrm{~ns}$, which is independent of the pulse separation between $2 \mathrm{~ns}$ to $4 \mathrm{~ns}$. These results indicate the existence of a very fast dephasing mechanism within $2 \mathrm{~ns}$.

The sample is a nitrogen delta-doped GaAs grown by metal organic chemical vapor deposition. The nitrogen sheet density is $2.9 \times 10^{12} \mathrm{~cm}^{-2}$. The details of the sample can be seen in Ref. 19. The sample was placed in a home-made confocal microscope system including a three-axis translational stage, which was inserted into an optical cryostat kept at $5 \mathrm{~K}$. Prior to the TPI measurements, we investigated three relevant parameters of the single nitrogen luminescence center: the multiphoton probability, the luminescence decay time $\mathrm{T}_{1}$, and the coherence time $\mathrm{T}_{2}$. Photon correlation measurements were carried out by using a Hanbury-Brown and Twiss (HBT) setup. $T_{1}$ and $T_{2}$ were measured by timecorrelated single photon counting and interferometric spectroscopy, respectively. A ps mode-locked Ti:sapphire laser operating at a repetition frequency of $82 \mathrm{MHz}$ was used to excite the sample at $815.8 \mathrm{~nm}$, which is slightly above the bandgap energy of GaAs at $5 \mathrm{~K}$. Pairs of picosecond pulses temporally separated by $\Delta$ were produced from the laser using an interferometer. PL from the sample was guided by a single-mode optical fiber to the outside of the cryostat, and a polarization controller was used to adjust the polarization of the output photons from the fiber. The PL signal was led to a spectrometer or another Michelson interferometer for $\mathrm{T}_{2}$ measurements, which was equipped with a high precision linear-motor stage. For TPI measurements, one of the arms of the interferometer was extended to convert to an 
asymmetric Michelson interferometer so as to compensate for the delay of the second photon, $\Delta .{ }^{11}$ A half-wave plate was inserted into one of the arms to vary the polarization condition to co-polarized or cross-polarized [see Fig. 2]. Except for the spectral measurements, the PL signal was spectrally filtered by a narrow bandpass filter (NBPF) with a spectral resolution of $400 \mu \mathrm{eV}(0.25 \mathrm{~nm})$. A pair of single photon detectors based on $\mathrm{Si}$ avalanche photodiodes was used in the HBT and TPI measurements. The same detector was also used in $T_{1}$ and $T_{2}$ measurements.

Figure 1(a) shows a typical PL spectrum of the sample at $5 \mathrm{~K}$ taken by using another optical microscope system with much lower spatial resolution. A bumpy luminescence band below $1508 \mathrm{meV}$ arises from the nitrogen impurity centers, which was first observed in our previous study ${ }^{19}$ and labeled NX centers in Ref. 27. Isolated nitrogen impurity has been shown experimentally to give a resonant defect level about $0.2 \mathrm{eV}$ above the conduction band minimum, ${ }^{28}$ and band anti-crossing models considering the repulsion between that level and the conduction band have been developed to explain the unusually large bandgap bowing of III-N-V alloys. ${ }^{29}$ On the other hand, some $\mathrm{N}$ complexes, including $\mathrm{NN}$ pairs,${ }^{30}$ are reported to have energy in the bandgap. We think that the NX center studied here is such an N complex. NX centers have a short radiative recombination time, typically less than $1 \mathrm{~ns}$, though they showed substantial inhomogeneity in their emission energy. ${ }^{19}$ The inset in Fig. 1(a)
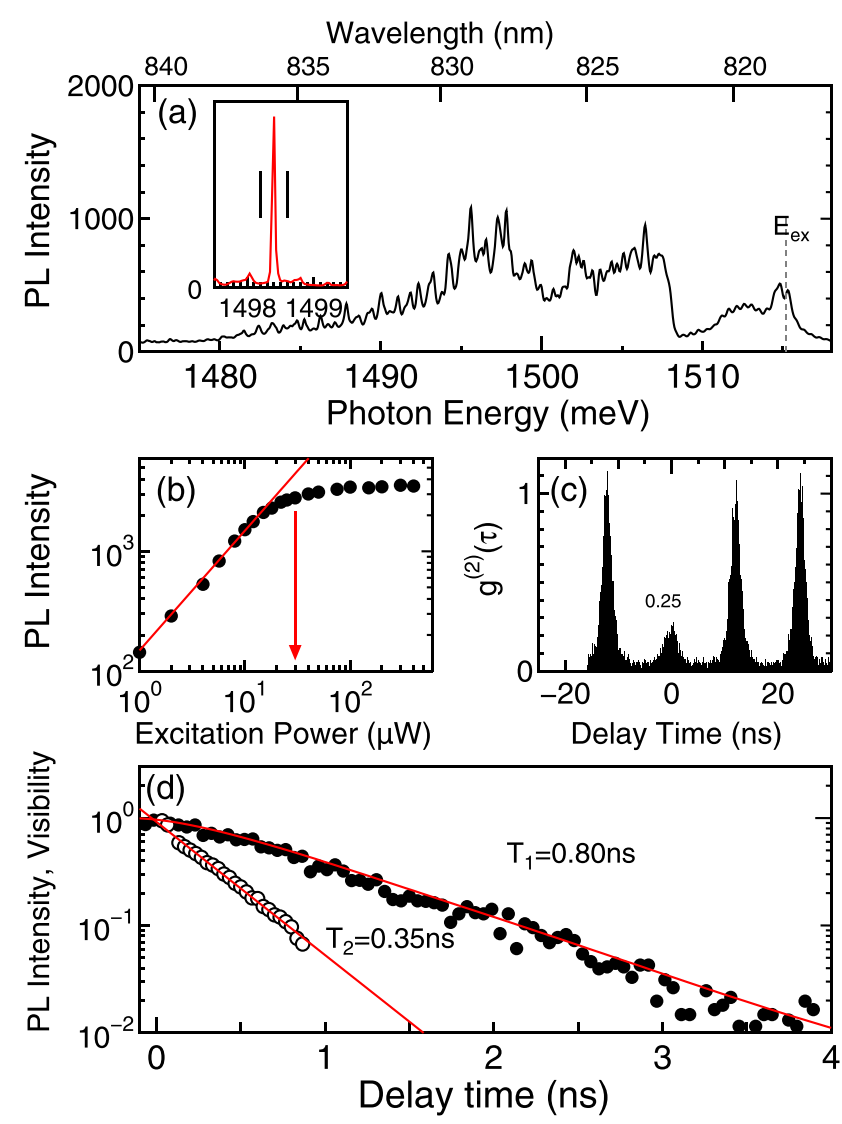

FIG. 1. (a) PL spectrum of the sample. The inset shows the PL spectrum of the single NX center used in this study. (b) Excitation power dependence of the PL intensity. The arrow indicates the excitation power used in the measurements. (c) The result of the HBT measurement on the center. (d) PL intensity (closed circles) and visibility (open circles) as a function of time. shows the PL spectrum of a single NX center used in the current study. The pass band of the NBPF is illustrated by two vertical lines. The PL spectrum of the single center was strongly linearly polarized along the [110] crystal axis and no orthogonally polarized components were observed. ${ }^{19}$ Figure 1(b) shows the excitation power dependence of the PL intensity of the center under pulse pair excitation with $\Delta=2 \mathrm{~ns}$. The solid line shows a linear fit for the data in the low power region. To ensure a high probability of single photon generation by an optical pulse, we intentionally carried out the TPI measurements under an excitation condition slightly exceeding the linear region. The excitation power used in the following measurements $(\sim 30 \mu \mathrm{W})$ is marked by the red arrow in the figure. It was confirmed that $\mathrm{T}_{2}$ does not strongly depend on the excitation power below this level.

Figure 1(c) shows the result of HBT measurements for the NX center, which was excited only once every $12.2 \mathrm{~ns}$ with an excitation power of $15 \mu \mathrm{W}$. A strongly reduced peak at a time delay of $0 \mathrm{~ns}$ is the signature for multiphoton suppression. The obtained $\mathrm{g}^{(2)}(0)$ was approximately 0.25 by taking the area of the central peak divided by the average area of the three side peaks. The PL decay curve was obtained under the same excitation conditions. The results are shown in Fig. 1(d) by the closed circles. After the convolution analysis with the instrument response function, the lifetime was determined to be $0.80 \mathrm{~ns}$. The dephasing time of the center was measured by scanning the position of a retroreflector in an arm of the Michelson interferometer. The fringe visibility is plotted in Fig. 1(d) by the open circles as a function of the delay time. The visibility decays as a single exponential with a decay time of $0.35 \mathrm{~ns}$. The obtained $\mathrm{T}_{2}$ does not reach the Fouriertransform limited value, $2 \mathrm{~T}_{1}$. A possible dephasing mechanism that is responsible for the shortened $T_{2}$ is the interaction with phonons. However, the contribution of phonons was found to be very limited at $5 \mathrm{~K}$ in our sample, which was clarified by a systematic study on the temperature dependence of $\mathrm{T}_{2}$ (details will be published elsewhere). Therefore, there must be another dephasing mechanism which is effective even at a low temperature. Spectral diffusion induced by a fluctuation of the electric field arising from the trapping and release of electric charges to traps near the emitter is often assumed.

The visibility of TPI can be simply estimated by $T_{2} /$ $2 T_{1} \cdot{ }^{31}$ By substituting the above-mentioned values of $\mathrm{T}_{1}$ and $\mathrm{T}_{2}$, we can estimate the visibility of TPI to be $22 \%$. Since the integration time for the $T_{2}$ measurement by interferometry is as long as seconds, all of the spectral diffusion processes, whose characteristic times range from nanosecond to seconds, can affect the results. However, if the dephasing mechanism is much slower than the time interval of the two photons, the experimentally obtained TPI visibility is expected to be higher than the estimated value. In such a case, its characteristic time can be determined from the $\Delta$ dependence of the TPI visibility. ${ }^{32}$

First, we measured the TPI from consecutive photons with a time interval of $2 \mathrm{~ns}$. The experiment was performed by using the optical system shown in the inset of Fig. 2(d). The path-length difference of the interferometer was properly adjusted to correspond to the time interval of the two excitation pulses, so that two consecutively emitted photons could reach the second beam splitter at the same time. The 

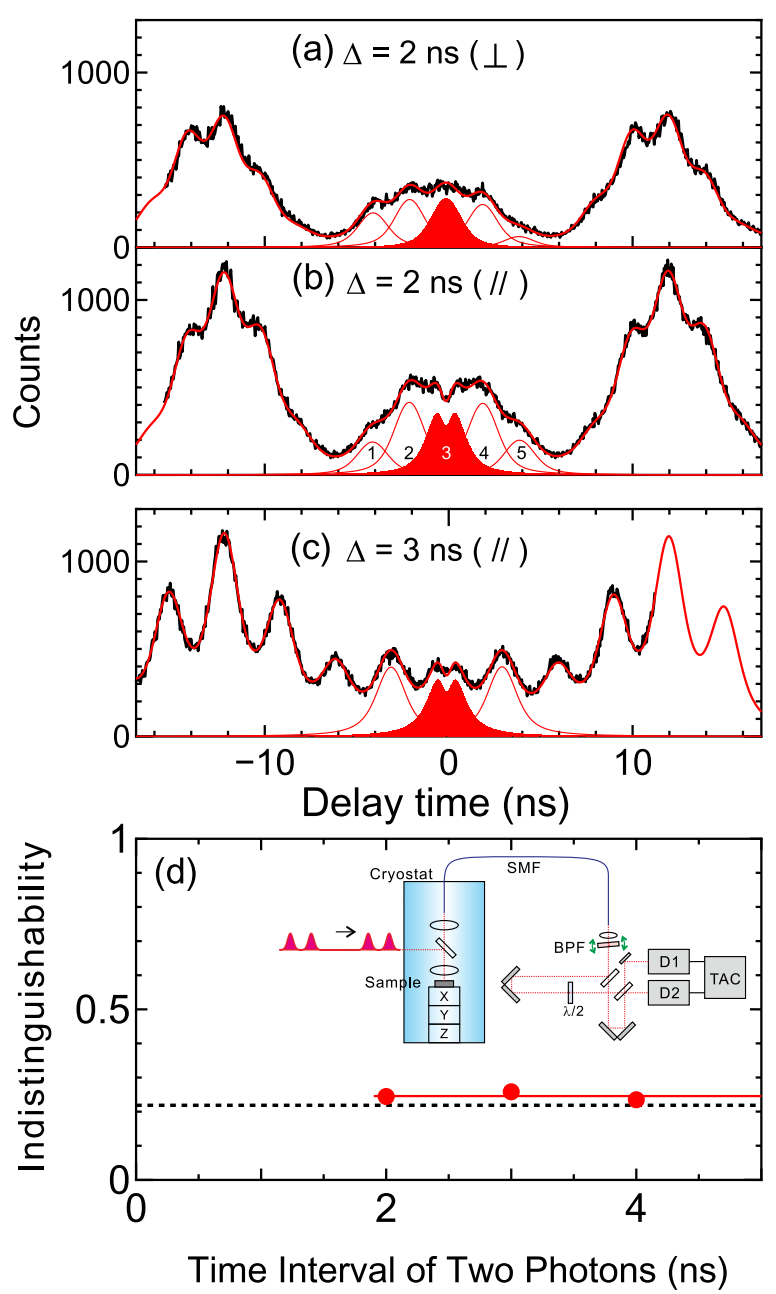

FIG. 2. Two-photon interference for the single nitrogen impurity center under a nonresonant excitation condition. (a) Two-photon interference with a cross-polarized direction and (b) co-polarized direction for $\Delta=2 \mathrm{~ns}$. The raw data are shown by the black solid line and the fitting results are shown by the red solid lines. (c) TPI for $\Delta=3 \mathrm{~ns}$. (d) Indistinguishability versus $\Delta$. The red dots are obtained from TPI measurements. The black dashed line shows the estimated value of $\mathrm{V}_{T P I}$ by $\mathrm{T}_{1}$ and $\mathrm{T}_{2}$.

correlation histograms of the consecutively emitted photons are shown in Fig. 2 for (a) cross-polarized and (b) copolarized configuration. Because of the finite radiative lifetime of the center, the five peaks composing the central cluster are not well separated. Therefore, the coincidence histograms were fitted by the sum of the Voigt functions which were separated by 2 ns. The fitting results are represented by thick and thin red curves. The asymmetric height of the histogram for cross-polarization arose from the deviation from a reflectivity:transmissivity $=1: 1$ of the beam splitter. The central peak is shown by the filled curves. In contrast to Fig. 2(a), a clear dip was observed for the central peak in Fig. 2(b), which unambiguously shows the TPI effect between two indistinguishable photons from a single nitrogen center.

To evaluate the degree of indistinguishability of photons, the following formula was used. ${ }^{11,33}$

$$
V=\left(\frac{1+2 g}{2(1+g)}-M\right) \frac{(1+g)\left(R T^{3}+R^{3} T\right)}{(1-\epsilon)^{2} T^{2} R^{2}},
$$

where $g$ is the multiphoton probability, and $\epsilon$ is the imperfection of the interferometer. $\mathrm{M}$ is given by $M=\frac{A_{3}}{A_{2}+A_{4}}$, where
$\mathrm{A}_{i}$ is the areas of the $i$-th peak, and $\mathrm{M}$ is 0.51 for the data in Fig. 2(b). This value is larger than 0.5 owing to nonzero $g$. We evaluated the imperfection of the asymmetric Michelson interferometer by using a ring cavity continuous wave (CW) Ti:Sapphire laser with very long coherence length. The fringe visibility was more than 0.9 regardless of the delay within the range of $\Delta$ used in this study, so the imperfection $\epsilon$ was less than 0.1 . Finally, the indistinguishability was calculated as 0.24 .

TPI measurements were carried out on two photons with time intervals of $3 \mathrm{~ns}$ and $4 \mathrm{~ns}$. As shown in Fig. 2(c), a clear dip can still be observed in the central peak. We plotted the $\mathrm{V}_{T P I}$ in Fig. 2(d). Of particular note is that, contrary to our expectation, the visibility did not depend on the pulse separation from $2 \mathrm{~ns}$ to $4 \mathrm{~ns}$, and the value of the constant visibility was almost the same as the estimated value based on $T_{1}$ and $\mathrm{T}_{2}$, as illustrated by the dotted line. These results suggest that the dephasing process responsible for a short $T_{2}$ is not slow, probably being faster than $2 \mathrm{~ns}$. Timing jitter in the photon emission process can also affect the two-photon indistinguishability. ${ }^{34}$ We estimated the reduction of $\mathrm{V}_{T P I}$ arising from the timing jitter based on the rise time of the $\mathrm{T}_{1}$ measurement data, and found that the reduction was not more than 0.11 . Therefore, this effect is not important for the point at issue.

Similar TPI measurements were reported recently in a single InGaAs QD. ${ }^{32}$ The authors observed $\Delta$-dependent $\mathrm{V}_{T P I}$ for $\Delta=2-12.5 \mathrm{~ns}$ under quasi-resonant excitation through the $p$-shell of the QD. For $\Delta=2 \mathrm{~ns}$, the two-photon visibility was as high as 0.94 for the neutral exciton peak at $7 \mathrm{~K}$. They attributed the observed $\Delta$-dependence to a nonMarkovian pure dephasing process, in particular, spectral diffusion caused by fluctuating charge traps in the vicinity of the QD. The process can be characterized by the correlation time $\tau_{c}$ and the amplitude. They reported a $\tau_{c}$ of approximately $12 \mathrm{~ns}$, which decreased rapidly with a temperature increase. The correlation time of such a process should depend on the nature of the material, and also the excitation conditions. In our case, the correlation time may be less than $2 \mathrm{~ns}$ even at $5 \mathrm{~K}$, as mentioned above. Therefore, we expect $\mathrm{V}_{T P I}$ to increase rapidly with decreasing $\Delta$ for $\Delta<2 \mathrm{ns,}$ though this is difficult to verify experimentally because of the finite radiative lifetime. One of the most likely causes of the different correlation time is the difference in the excitation method. In contrast to their experiments, where a quasiresonant $p$-shell excitation was used, nonresonant above gap excitation was used in our case. This means that a relatively high density of free carriers was created instantaneously in GaAs. This may be related to the fast dephasing process within $2 \mathrm{~ns}$. At present, we have not determined the specific mechanism of such a fast dephasing process, but the smallness ( $\sim 15 \mathrm{meV})$ of the energy difference between the bound exciton state (NX center) and continuum state (bandgap of GaAs) compared with a typical QD $(\sim 100 \mathrm{meV})$ may be crucial for the fast dephasing. As reported in many QDs, quasiresonant excitation can strongly improve the two-photon visibility $^{14}$ compared with nonresonant excitation, and weak optical excitation above the bandgap can further improve the visibility in some cases. ${ }^{35} \mathrm{We}$ expect that the resonant excitation can reduce the free carrier responsible for the fast 
dephasing, and greatly increases the two-photon visibility of a nitrogen isoelectronic impurity center in GaAs. Moreover, as discussed in Ref. 32, carriers in the wetting layer in a QD sample affect $\mathrm{V}_{T P I}$ at temperatures above $30 \mathrm{~K}$. In such a process, the spatial distribution of traps near the emitter, as well as the number of traps, should be important for the dephasing rate. Unlike typical Stranski-Krastanov QDs with wetting layer, there is no wetting layer in our nitrogen deltadoped GaAs, and consequently, the luminescence center will be affected from traps or carriers from all directions around the luminescent center. Such differences may be related to the large difference in the correlation time mentioned above.

As described above, we have shown that the fast dephasing mechanism governs $\mathrm{V}_{T P I}$ under nonresonant pulsed excitation. However, this does not mean that there is no slow spectral diffusion in our sample. In this context, it should be mentioned that we have observed a shorter coherence time $\left(\mathrm{T}_{2}=305 \mathrm{ps}\right)$ under $\mathrm{CW}$ excitation at the same excitation wavelength and with even lower average excitation power than the pulsed excitation. This arises from a slow spectral diffusion. Charge carriers can be replenished continuously in the CW excitation scheme, which may induce slow spectral diffusion by fluctuating charge traps during an accumulation time of seconds. Since the density of the initially generated carriers should be greater in pulsed excitation, the carriers may occupy almost all trap sites in the vicinity of the luminescence center and, as a result, the electric field felt by the luminescence center may be kept constant during approximately $\mathrm{T}_{1}$ for every pulse, and the energy of the emitted photon does not fluctuate.

In summary, we have investigated the indistinguishability of photons emitted from a single nitrogen impurity center in GaAs under a nonresonant excitation scheme. For the TPI of the two photons with a time interval of $2 \mathrm{~ns}$ to $4 \mathrm{~ns}$, the degree of indistinguishability has almost the same value of approximately 0.24 . These results indicate the existence of a very fast dephasing process within $2 \mathrm{~ns}$, which may be related to the instantaneously generated free carriers in GaAs. We believe that our demonstration is an essential step toward future quantum information processing using impurities in III-V compound semiconductors.

This work was supported by the JSPS KAKENHI Grant No. JP25289091, Research Foundation for Opto-Science and Technology, and SEI Group CSR Foundation.

${ }^{1}$ C. H. Bennett, G. Brassard, R. Jozsa, A. Peres, and W. K. Wootters, Phys. Rev. Lett. 70, 1895 (1993).

${ }^{2}$ D. Bouwmeester, J.-W. Pan, K. Mattle, M. Eibl, H. Weinfurter, and A. Zeilinger, Nature 390, 575 (1997).

${ }^{3}$ H. de Riedmatten, I. Marcikic, W. Tittel, H. Zbinden, D. Collins, and N. Gisin, Phys. Rev. Lett. 92, 047904 (2004).

${ }^{4}$ E. Knill, R. Laflamme, and G. J. Milburn, Nature 409, 46 (2001).
${ }^{5}$ P. Kok, K. Nemoto, T. C. Ralph, J. P. Dowling, and G. J. Milburn, Reviews of Modern Physics 79, 135 (2007).

${ }^{6}$ J. Beugnon, M. P. A. Jones, J. Dingjan, B. Darquié, G. Messin, A. Browaeys, and P. Grangier, Nature 440, 779 (2006).

${ }^{7}$ P. Maunz, D. L. Moehring, S. Olmschenk, K. C. Younge, D. N. Matsukevich, and C. Monroe, Nat. Phys. 3, 538 (2007).

${ }^{8}$ A. Kiraz, M. Ehrl, T. Hellerer, Ö. E. Müstecaplioglu, C. Bräuchle, and A. Zumbusch, Phys. Rev. Lett. 94, 223602 (2005).

${ }^{9}$ H. Bernien, L. Childress, L. Robledo, M. Markham, D. Twitchen, and R. Hanson, Phys. Rev. Lett. 108, 043604 (2012).

${ }^{10}$ A. Sipahigil, M. Goldman, E. Togan, Y. Chu, M. Markham, D. Twitchen, A. Zibrov, A. Kubanek, and M. Lukin, Phys. Rev. Lett. 108, 143601 (2012).

${ }^{11}$ C. Santori, D. Fattal, J. Vucković, G. S. Solomon, and Y. Yamamoto, Nature 419, 594 (2002).

${ }^{12}$ Y.-j. Wei, Y.-M. He, M.-C. Chen, Y.-N. Hu, Y. He, D. Wu, C. Schneider, M. Kamp, S. Höfling, C.-Y. Lu, and J.-W. Pan, Nano Lett. 14, 6515 (2014).

${ }^{13}$ R. Patel, A. Bennett, K. Cooper, P. Atkinson, C. Nicoll, D. Ritchie, and A. Shields, Phys. Rev. Lett. 100, 207405 (2008).

${ }^{14}$ P. Gold, A. Thoma, S. Maier, S. Reitzenstein, C. Schneider, S. Höfling, and M. Kamp, Phys. Rev. B 89, 035313 (2014).

${ }^{15}$ E. B. Flagg, A. Muller, S. V. Polyakov, A. Ling, A. Migdall, and G. S. Solomon, Phys. Rev. Lett. 104, 137401 (2010).

${ }^{16}$ R. B. Patel, A. J. Bennett, I. Farrer, C. A. Nicoll, D. A. Ritchie, and A. J. Shields, Nat. Photon. 4, 632 (2010).

${ }^{17}$ K. Sanaka, A. Pawlis, T. D. Ladd, K. Lischka, and Y. Yamamoto, Phys. Rev. Lett. 103, 053601 (2009).

${ }^{18}$ M. Ikezawa, Y. Sakuma, and Y. Masumoto, Jpn. J. Appl. Phys. 46, L871 (2007).

${ }^{19}$ M. Ikezawa, Y. Sakuma, L. Zhang, Y. Sone, T. Mori, T. Hamano, M. Watanabe, K. Sakoda, and Y. Masumoto, Appl. Phys. Lett. 100, 042106 (2012).

${ }^{20}$ L. Zhang, M. Ikezawa, T. Mori, S. Umehara, Y. Sakuma, K. Sakoda, and Y. Masumoto, Jpn. J. Appl. Phys., Part 1 52, 04CG11 (2013).

${ }^{21}$ D. G. Thomas, J. J. Hopfield, and C. Frosch, Phys. Rev. Lett. 15, 857 (1965).

${ }^{22}$ R. Schwabe, W. Seifert, F. Bugge, R. Bindemann, V. F. Agekyan, and S. V. Pogarev, Solid State Commun. 55, 167 (1985).

${ }^{23}$ T. Makimoto and N. Kobayashi, Appl. Phys. Lett. 67, 688 (1995).

${ }^{24}$ S. Francoeur, J. F. Klem, and A. Mascarenhas, Phys. Rev. Lett. 93, 067403 (2004).

${ }^{25}$ T. Kita and O. Wada, Phys. Rev. B 74, 035213 (2006).

${ }^{26}$ Y. Endo, Y. Hijikata, H. Yaguchi, S. Yoshida, M. Yoshita, H. Akiyama, F. Nakajima, R. Katayama, and K. Onabe, Physica E 40, 2110 (2008).

${ }^{27}$ Y. Sakuma, M. Ikezawa, and L. Zhang, in Micro- and Nanophotonic Technologies, edited by P. Meyrueis, K. Sakoda, and M. Van de Voorde (Wiley-VCH, Weinheim, 2017) pp. 125-141.

${ }^{28}$ D. J. Wolford, J. A. Bradley, K. Fry, and J. Thompson, in Proceedings of the 17th International Conference on the Physics of Semiconductors, edited by J. D. Chadi and W. A. Harrison (Springer-Verlag, New York, 1984) pp. 627-630.

${ }^{29}$ W. Shan, W. Walukiewicz, J. Ager, E. Haller, J. Geisz, D. Friedman, J. Olson, and S. Kurtz, Phys. Rev. Lett. 82, 1221 (1999).

${ }^{30}$ X. Liu, M. E. Pistol, L. Samuelson, S. Schwetlick, and W. Seifert, Appl. Phys. Lett. 56, 1451 (1990).

${ }^{31}$ J. Bylander, I. Robert-Philip, and I. Abram, Eur. Phys. J. D 22, 295 (2003).

${ }^{32}$ A. Thoma, P. Schnauber, M. Gschrey, M. Seifried, J. Wolters, J.-H. Schulze, A. Strittmatter, S. Rodt, A. Carmele, A. Knorr, T. Heindel, and S. Reitzenstein, Phys. Rev. Lett. 116, 033601 (2016).

${ }^{33}$ S. Ates, I. Agha, A. Gulinatti, I. Rech, A. Badolato, and K. Srinivasan, Sci. Rep. 3, 1397 (2013).

${ }^{34}$ A. Kiraz, M. Atatüre, and A. Imamoglu, Phys. Rev. A 69, 032305 (2004).

${ }^{35}$ O. Gazzano, S. M. de Vasconcellos, C. Arnold, A. Nowak, E. Galopin, I. Sagnes, L. Lanco, A. Lemaître, and P. Senellart, Nat. Commun. 4, 1425 (2013). 